\title{
Social robots: The influence of human and robot characteristics on acceptance
}

https://doi.org/10.1515/pjbr-2019-0028

Received May 2, 2019; accepted September 12, 2019

\begin{abstract}
Research in social robotics is focused on the development of robots that can provide physical and cognitive support in a socially interactive way. Whilst some studies have previously investigated the importance of user characteristics (age, gender, education, robot familiarity, mood) in the acceptance of social robots as well as the influence a robot's displayed emotion (positive, negative, neutral) has on the interaction, these two aspects are rarely combined. Therefore, this study attempts to highlight the need to consider the influence that both human and robot attributes can have on social robot acceptance. Eighty-six participants completed implicit and explicit measures of mood before viewing one of three video clips containing a positive, negative or neutral social robot (Pepper) followed by questionnaires on robot acceptance and perception. Gender and education were not associated with acceptance; however, several constructs of the acceptance questionnaire significantly correlated with age and mood. For example, those younger and those experiencing sadness or loneliness were more dependent on the opinions of others (as measured by the social influence construct of the acceptance questionnaire). This highlights the importance of mood in the introduction of social robots into vulnerable populations. Robot familiarity also correlated with robot acceptance with those more familiar finding the robot less useful and less enjoyable, this is important as robots become more prominent in society. Displayed robot emotion significantly influenced acceptance and perception with the positive robot appearing more childlike than the negative and neutral robot, and the neutral robot the least helpful. These findings emphasise the importance of both user and robot characteristics in the successful integration of social robots.
\end{abstract}

Keywords: social robots, robot acceptance, human-robot interaction, artificial emotion, affect

\footnotetext{
*Corresponding Author: Laura Bishop: Cardiff University, Cardiff, UK; E-mail: bishoplm2@cardiff.ac.uk
}

\section{Introduction}

In recent years the field of social robotics has expanded and is now a growing topic of public interest and concern. In the last few decades the tasks performed by robots have been largely repetitive or dangerous jobs that humans are less willing or able to do, such as work on assembly-lines within factories or bomb disposal in the military [1]. However, with people now living longer and experiencing career pressures and health issues [2], focus is expanding to include assistive robots (AR) within the home and clinical settings. AR are defined as robots that provide physical or cognitive support to a human user and can help with activities of daily living, rehabilitation exercises, physical assistance and so forth.

There is also an increasing demand for robots to be socially interactive helping improve the safety, wellbeing and independence of the user whilst reducing the stress and concern felt by their family and friends [3]. An example of where social robot assistance can be employed is in the case of social isolation, something often experienced when mobility has decreased and the maintenance of social networks has become more difficult. Social isolation can negatively impact cognitive functioning, levels of physical activity and even mortality with social robots helping alleviate these concerns [4]. Socially assistive robots (SAR) provide assistance through social interaction [5], interpreting environments and responding in a natural and human-like way [6], whilst providing physical assistance, cognitive stimulation, health management and psychosocial support [7].

Populations that can benefit from the use of SAR include older adults, individuals with physical or cognitive impairments and those in convalescent care [5]. SAR are already being utilised with stroke patients, weight-loss programmes and patient education [8], with the number and types of tasks undertaken increasing as technology ad-

Anouk van Maris, Sanja Dogramadzi: Bristol Robotics Laboratory, University of the West of England, Bristol, UK

Nancy Zook: University of the West of England, Bristol, UK 
vances. It is not however only technology that will determine the tasks undertaken by social robots but also what assistance humans are willing to accept. Current research suggests people are happier utilising robots in tasks that require low interaction, such as cleaning, but are less accepting of robots performing tasks that require high interaction such as administering personal care [9], [10]. Therefore, investigating acceptance is extremely important when considering SAR for tasks that require high interaction such as companionship in the case of social isolation.

The aim of this study was to determine whether specific user characteristics were related to social robot acceptance. In addition, robot appearance and behaviour were also investigated using the humanoid robot Pepper [https://www.softbankrobotics.com/emea/en/pepper] to identify if these factors were related to a more positive perception of the technology. Understanding factors associated with robot acceptance will inform future research and design of socially assistive robots.

\section{Background}

The field of social robotics has experienced rapid change over the last few decades, with increased focus on robots that can integrate into human environments in order to interactively provide humans physical and cognitive assistance. In order for robots to be accepted it is important to not only consider how the robot's capabilities influence acceptance but also how the characteristics of the human user relates to the interaction. By better understanding what factors influence social robot acceptance, considerations can be made when designing and integrating robots to improve both levels of acceptance and the human-robot interaction more generally.

\subsection{Human acceptance of social robots}

Whilst socially assistive robots offer great benefits, a certain level of robot acceptance is required to ensure individuals will actually use them. Acceptance can be defined as the positive evaluation of a robot that results in the intention and eventual act of using the technology [11]. The Technology Acceptance Model (TAM) suggests that the main influencers of acceptance are how useful the technology will be in enhancing user performance and how potentially effortless it is to use (Davis, 1985). The TAM has been further extended by the Unified Theory of Acceptance and Use of Technology model (UTAUT) that also identifies the opinion of others and a good supporting infrastructure as factors influencing user acceptance [12]. Taken together these models suggest acceptance to be based on how easy the technology is to use, how useful it is perceived to be, whether it has a facilitating infrastructure and if others agree that the technology should be employed. These models do not however consider the social aspect of the human-robot interaction that becomes increasingly important as humans and robots start to work more closely together [13].

The Almere Model of Acceptance, adapted from UTAUT, posits that the human-robot interaction is as important to acceptance as the more functional components and includes factors such as whether the robot is viewed as capable of social interaction and whether it will bring feelings of enjoyment when using it [14]. The model was however created specifically for use within the older adult population and requires further analysis to determine its reliability more generally. If psychometrically sound, the questionnaire would be used to determine whether factors related to both the user and the robot can be predictors of robot acceptance.

\subsection{User characteristics and social robot acceptance}

There are a number of user characteristics that have been previously found to influence robot acceptance and should therefore be considered when looking to integrate SAR into homes and care homes. By understanding how user characteristics relate to robot acceptance roboticists can better understand the qualities social robots require, and how they may need to be adapted to individual users. These include age, gender, education and a user's previous experience with technology [15]. However, as these findings were within an older adult population there is a need for further investigation within other age groups.

In relation to age, older participants were found to experience more anxiety around using a robot likely impacting robot acceptance [15]. This study was however limited to adults aged 65 years plus with research later extending the age range to 40 years plus finding no significant results [16]. It must therefore be considered whether the increasing anxiety felt by those over the age of 65 is due to a cohort effect that dissipates as younger generations become more familiar with technology. It has also been found that human anxiety correlates with gender and level of education, where women experience more anxiety than men, and anxiety increases as level of education decreases [17], 
[15]. Women and older adults are also less willing to utilise robots, with men more likely to hold positive views of the technology [18], [15], [16]. This is however dependent on robot type with women more accepting of robot pets than those with anthropomorphic, lifelike qualities [17].

In relation to robot familiarity, previous technological experience has been found to result in less robot anxiety, an increase in perceived safety and more confidence in facilitating conditions [15]. Robot familiarity is also said to result in a robot appearing less intelligent [19], possibly due to a clearer understanding of the current limitations of robot autonomy and capabilities. This study looked to extend previous research by analysing user characteristics across an expanded age range, providing a more comprehensive overview of what roboticists need to consider when creating social robots, and the barriers developers should consider when looking to integrate social robots into home or clinical settings.

\subsection{User mood and social robot acceptance}

Users of socially assistive robots will often be from vulnerable populations such as older adults, children and the mentally or physically unwell, all of which may regularly experience low mood such as depression. Mood is an important aspect of social interaction, with people often misinterpreting their current mood as a reflection of how they feel about the people and objects around them [20]. Research specifically focused on how user mood relates to robot acceptance is limited; however a study by Baisch et al. [21] found life satisfaction to positively correlate with intention to use a robot, with depressive mood having no relationship. Baisch et al's study did not utilise a robot with a human morphology and different results may be found using a more anthropomorphic robot. A relationship between user mood and acceptance could be problematic should a social robot be assigned to increase the general well-being of somebody currently experiencing low mood. Mood can have a direct impact on higher-order cognitive functioning such as judgement, interpretation, decision making and reasoning. All of these are highly important during the evaluation of surrounding situations [22].

Mood is defined as relatively long lasting feelings of affect that cannot generally be related to a specific situation or event [23]. It runs along the axis of two dominant dimensions, positive affect and negative affect, with positive affect ranging from a sense of alertness to lethargy and negative affect from distressed to calm [24]. The Positive and Negative affect scale (PANAS-X;[25]) is a self-rated affect scale developed to measure these two dimensions of mood. Explicit mood ratings can however be susceptible to social desirability as participants may not be comfortable revealing their true feelings. Therefore implicit affect measures such as The Implicit Positive and Negative Affect Test (IPANAT; [26]) have been developed. These measure impulsive rather than reflexive affect by relying on the way in which humans will project their more immediate mood onto the things around them. Without considering how user mood influences robot acceptance, there may be potential limits to the benefits of SAR within vulnerable populations. Based on this, investigating how both implicit and explicit mood relates to constructs found within the Almere model of Acceptance will highlight factors that require consideration before social robot integration.

\subsection{Robot displayed emotion and acceptance}

In addition to understanding how user emotion relates to the acceptance of social robots, the expression of emotion by the robot itself could also influence the interaction. An important aspect of any social communication is the emotional exchange between individuals, each interpreting the other's feelings, understanding their intention and reacting in a complementary way [27]. Suppressing emotion during a dyadic interaction blocks this transmission and therefore the ability to acknowledge and respond to each other's feelings. This barrier can impact both the quality of the communication as well as the ability to form a bond [28]. Therefore, whilst improving the functionality of social robots is essential, it is also fundamental that the robot is able to communicate in a way that is seen as socially acceptable [6].

A study by De Graaf, Allouch and Van Dijk [6] found a robot's ability to hold dialogue, analyse their user's current mood and express emotion as the key characteristics required for a social robot to be accepted. It is also more important for the emotion expressed by a robot to be otheroriented rather than self-motivated, resulting in a robot that is perceived as caring, likeable and trustworthy and more friendly $[29,30]$. Empathy is the ability to understand the current emotional state of another and respond in an affective way [31]. In a medical or home care setting empathy and sympathy are at the base of social etiquette and require the expression of both positive and negative emotions [32]. It is therefore important to understand the specific influences that negative, as well as neutral and positive emotions have on a human-robot interaction. This research looked to investigate whether a robot portraying 
basic contextual emotions significantly influences human acceptance of social robots.

\subsection{Human perception of social robots}

Whilst robot acceptance is a key indicator of human attitudes and behaviours, it is also important to understand how the technology is more generally perceived. Upon initially meeting someone, humans will assign traits and stereotypes to that person based on their physical attributes and overt behaviours [33]. These expectations will be in line with a person's previous experiences and results in judgements on how they anticipate someone to behave [34]. As with human-human contact, a user will likely attribute traits to a robot based solely on its appearance and observable behaviours [35].

With socially assistive robots undertaking a wide range of roles it is crucial to understand which specific robot characteristics influence how the robot is perceived. Literature to date states the importance of matching a robot to its tasks, with users making assumptions on the robot's capabilities even prior to first use [36], [37]. SAR may therefore require a human-like, anthropomorphic embodiment and personality in order to appear capable of performing tasks that involve high-level social interaction [36]. Anthropomorphism may be necessary for robots to appear socially capable, but also to enable them to move around in a world built for the human body [38]. However, should a socially assistive robot be built to appear more anthropomorphic than its capabilities, disappointment may arise as soon as expectations are not met [39], [40].

The Godspeed Questionnaire [39] provides a measurement of how human-like, lifelike, likeable, intelligent and safe a robot is perceived to be in relation to the characteristics it portrays. Should a robot be rated high on these qualities it is assumed that the robot is positively perceived resulting in positive behaviours towards the technology. By analysing how both human characteristics and robot emotion can influence robot perception, as well as how the questionnaire relates to the constructs included in the Almere model of Acceptance, a sound overview of how human and robot factors influence the human-robot interaction can be developed.

Interest in social robots is rising with the need to investigate factors that can influence their successful integration becoming more crucial. It is highly important when considering these factors that both characteristics within the user, and elements built into the robot are considered. This study firstly tested the internal consistency of the Almere model questionnaire when extended outside of its previous work with older adults, ensuring its reliability before being utilised. It then investigated how participant characteristics (age, gender, level of education, robot familiarity) relate to robot acceptance, with previous work by Heerink [15] supporting the prediction that significant correlations would be found between each of these characteristics and participant acceptance ratings. User mood was also investigated to determine whether it significantly relates to robot acceptance. Whilst research in this area is limited, work by Baisch et al. [41] does indicate a relationship between user well-being and acceptance, and research in human-human relations suggests mood to impact an interaction more generally [20]. It was therefore predicted that user mood would significantly correlate with acceptance of a social robot. Investigations then took place to determine whether a robot displaying either positive, negative or neutral emotion while reading a news article would also influence user acceptance. Although not directly comparable, previous research indicated that displays of emotion by a robot can influence how that robot is perceived [29]. It was therefore hypothesised that acceptance levels would significantly differ depending on the robot emotion being displayed. It was also predicted that a user's acceptance of a positive, negative or neutral robot would differ depending on whether participants are feeling happy or sad.

To summarise, this study predicts user characteristics (age, gender, education, robot familiarity, mood) and robot displayed emotion (positive, negative, neutral) to be important factors in the acceptance of social robots. Improving understanding in this area will help highlight the need for roboticists to consider the complexity of the human-robot interaction and how both human and robot characteristics should be considered.

\section{Method}

\subsection{Participants}

Eighty-six participants were recruited through the University of the West of England (UWE) Psychology Student Participant Pool as well as via social media advertising. Participants were randomly selected to experience one of three experimental levels viewing a short clip (around 35 seconds) of a social robo t displaying either neutral $(N=45)$, positive $(N=20)$ or negative $(N=$ 21) contextual emotion. More participants were selected for the neutral experimental level in order to effectively 
analyse the validity of the Almere questionnaire as well as user characteristics in relation to acceptance. Participants were exposed to all other measures within the study. Of the 86 participants 20 were male, 66 female ranging from 18 to 72 years $(M=29.26, S D=14.98)$. The sample was well educated (rated from GCSEs to doctorate level) with 71 of the 86 participants studying or in receipt of an undergraduate degree or above. For the neutral sample 13 were male, 32 female (age $M=30.79, S D=16.53$ ). For the positive sample 4 were male, 16 female (age $M=30.85, S D$ $=16.93)$. For the negative sample 3 were male, 18 female (age $M=25.19, S D=8.35$ ).

\subsection{Study design and procedure}

The study employed a between-subject design to investigate how user characteristics (age, gender, education, robot familiarity) and participant mood (implicit, explicit) relate to social robot acceptance and perception and whether the influence of a robot's displayed contextual emotion (neutral, positive, negative) can also influence acceptance and perception. A 2 (sad/happy) x 3 (neutral/positive/negative) factorial design was then used to investigate the interaction between user mood and displayed robot emotion and its impact on robot acceptance and perception. Participants were placed into 'sad' $(N=$ 21) and 'happy' $(N=21)$ groups utilising the sub-categories found within the PANAS-X, with those having experienced 'sad' feelings 'quite a bit' or 'extremely' during the past few weeks placed into the 'sad' group (low positive affect, high negative affect) and those who on average had experienced these feelings 'very slightly or not at all' or 'a little' were placed into the 'happy' group (high positive affect, low negative affect). Those who on average experienced these feelings 'moderately' were not included in the analysis.

Participants accessed the study through the online Qualtrics ${ }^{\circledR}$ survey platform on PCs or tablets. After being provided with a brief introduction and request for consent participants were asked to record their age, gender and level of education as well as answer the question 'Please rate your familiarity with robots' on a scale of 1 (very familiar) to 5 (not at all familiar). Implicit mood was then recorded using the iPANAT [26], presenting participants with six non-existing words to which they were asked to rate how happy, helpless, energetic, tense, cheerful and inhibited the word sounds on a scale of 1 (doesn't fit at all) to 5 (fits very well). Explicit mood was then measured through the PANAS-X [25] with participants rating 60 emotion words (e.g. cheerful) depending on the extent to which they had felt the emotion in the past few weeks. This was again measured through a 5 point Likert-type scale ranging from 1 (very slightly or not at all) to 5 (extremely), with words from both measures presented to participants in random order.

Participants were then instructed that a video would shortly play containing a social robot with a brief description of a social robot's role. Each video contained the social robot Pepper telling one of three fictitious news stories about polar bears in a contextually neutral, happy or sad way. The negative story contained details around the effects of global warming on polar bears, the positive story focused on a mother and cub playing in the snow, and the neutral story supplied general polar bear facts. The software used to program Pepper was Choregraphe 2.5.5 with the emotive behaviours implemented based on literature by Beck et al. [42] and Kwon et al. [43]. Pepper's emotions were distinguished by head position, pitch and speed of voice; with happy behaviour including a raised chin, high pitch and speed of speech and more extreme movements. Sad behaviour included a lowered chin, a low pitch and speed of speech, and small movements with neutral an average of the previous two. These behaviours were tested through an online survey that found participants significantly able to distinguish between the sad, happy and neutral behaviours when presented non-contextually [44]. Example snippets of these behaviours are shown in Fig. 1.

After the video had finished acceptance was measured using the Almere model questionnaire [14] containing 41 questions under 12 overarching constructs (e.g. perceived ease of use). Participants were asked to rate how much they agreed with each statement from 1 (totally disagree) to 5 (totally agree). As the questionnaire was originally designed for use within the older adult population and with longer exposure in mind, a number of the questions required slight alteration such as 'I plan to use the robot during the next few days' changed to 'I would plan to use the robot during the next few days'. Finally each participant completed the Godspeed questionnaire of robot perception [39] that includes a series of fives questionnaires on anthropomorphism, animacy, likeability, perceived intelligence and perceived safety containing a total of 24 semantic differential scales. After completion of all questionnaires participants were provided with a study debrief and assigned a study credit if applicable. The study took around 30 minutes for participants to complete. 


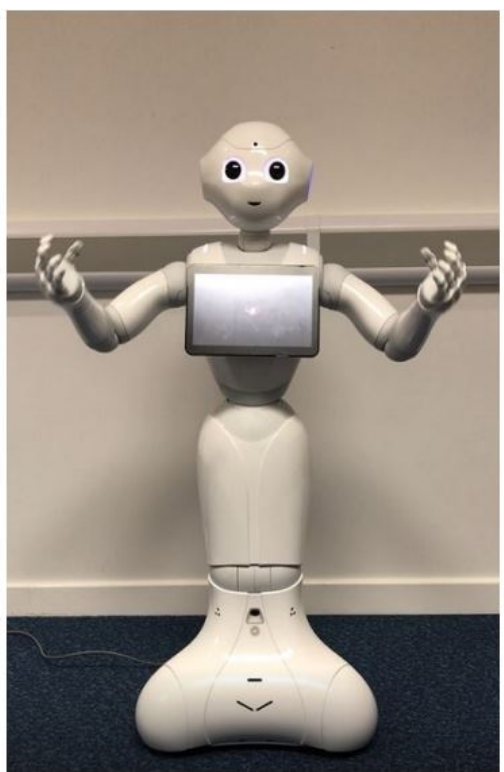

(a)

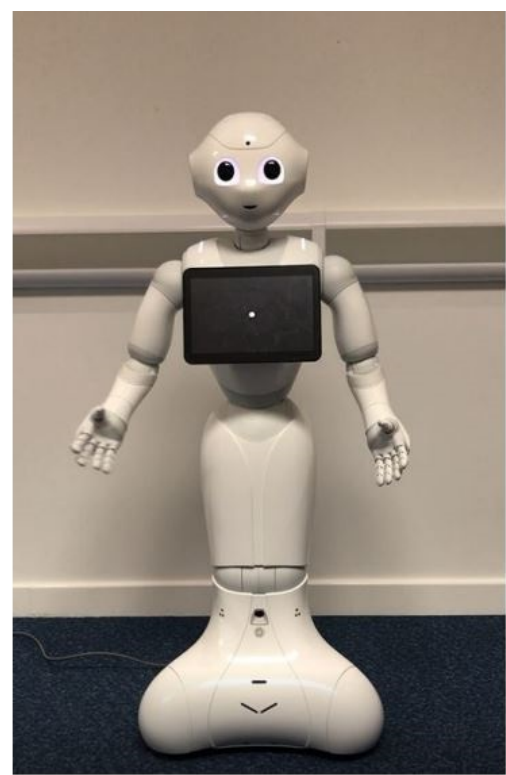

(b)

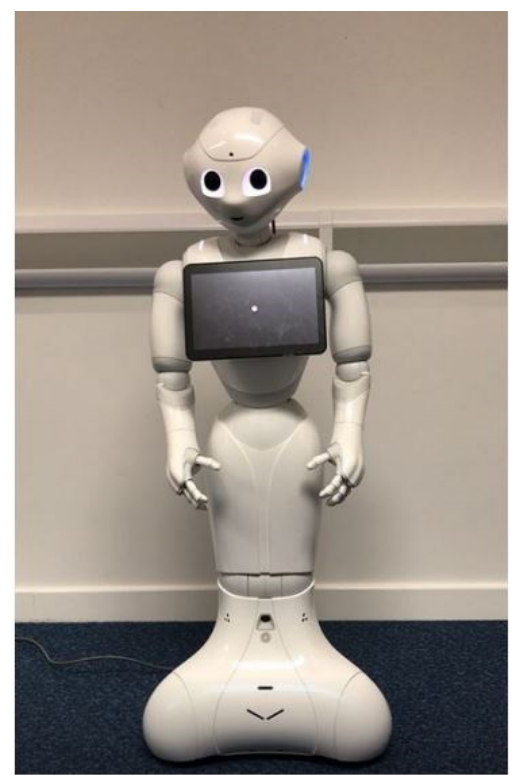

(c)

Figure 1: Example snippets of happy (a), neutral (b) and sad (c) robot behaviours.

\section{Results}

This study investigated how social robot acceptance and the way in which a robot is perceived can be influenced by both the characteristics of the user and the attributes of the robot. The human characteristics analysed were age, gender, education, robot familiarity and user mood as well as the displayed emotion of the robot itself (neutral/happy/sad). Also investigated was te relationship between the acceptance measure (Almere Model) and the robot perception measure (Godspeed Questionnaire) in order to determine any potential correlations, as well as a test of internal consistency to determine whether the Almere Model of Acceptance could be extended beyond the older adult population for which it was originally designed. The key assumptions for parametric testing were met with only a slight skew within the facilitating conditions construct of the Almere model and the perceived safety construct within the Godspeed model. As a result of this and previous research into these measures largely utilsing parametric testing, the same has been applied within this paper.

\subsection{User characteristics and social robot acceptance}

The Almere Model's 12 construct questionnaire has previously reported acceptable reliability with a Cronbach's al- pha of .7 when used within the older adult population [14], [15]. An analysis of reliability was therefore conducted to establish whether the Almere model questionnaire maintained internal consistency when extended across a larger age range. Cronbach's alpha reached high reliability $(\alpha=$ .86). A Cronbach's alpha reliability analysis was also carried out on the Godspeed questionnaire again with high internal consistency $(\alpha=.90)$ and only one semantic differentiation scale, Quiescent: Surprised, worth considering for removal increasing alpha to $\alpha=.91$.

Correlation analyses were then conducted to investigate potential relationships between user characteristics and robot acceptance using Pearson's Correlation Coefficient to determine both significance and effect size. It is important to note that due to the minimal effort required to complete an online study differences between those participants compensated and those not compensated were not taken into consideration during analysis. Age was firstly investigated in relation to the 12 constructs included in the Almere questionnaire with moderate negative correlations found between age and the constructs of perceived enjoyment of the robot $(r=-0.38, n=45, p=.01)$ and social influence $(r=-0.30, n=45, p=.05)$. The younger the participants, the more pleasure they anticipated when using the robot. The younger participants relied more heavily on the opinions of others when deciding whether to utilise it. All other constructs within the acceptance questionnaire were not found to significantly correlate with age. When analysing the relationship between age and the five con- 
structs of perception within the Godspeed questionnaire no significant correlations were found.

In relation to gender, a one-way analysis of variance found no significant differences between men and women in any of the 12 constructs within the Almere acceptance questionnaire or the Godspeed questionnaire of robot perception. An additional one-way analysis of variance investigated differences between a participant's level of education and acceptance, with the only significant difference found in the construct of perceived usefulness $(F(4,40)=$ $2.73, p=.04, R^{2}=.22$ ). Those in receipt of a doctorate qualification rated the robot as significantly less useful than other levels of education; however, with only two participants present in this group this result is not meaningful. No significant differences were found between education and the way in which the robot was perceived. A correlation analysis was also conducted between participant education and acceptance with no significant relationships found.

In respect of robot familiarity, the largest proportion of participants rated themselves as either 'somewhat familiar' (39.5\%), or 'not very familiar' (32.6\%), with no participants indicating they were 'very familiar' (see Table 1).

In order to investigate the relationship between robot familiarity and acceptance, correlation analyses were conducted finding strong negative relationships between robot familiarity and both facilitating conditions $(r=-0.50$, $n=45, p<.01)$ and perceived robot safety $(r=-0.52, n=45, p$ $<.001)$. Participants more familiar with robots felt they had less sufficient knowledge and tools required to use the system and found the robot less safe. Moderate negative relationships were also found between robot familiarity and intention to use $(r=-0.40)$, perceived adaptiveness $(r=-$ $0.36)$, perceived usefulness $(r=-0.38)$ and perceived enjoyment in using the robot $(r=-0.37)$ with those more familiar rating themselves as less likely to use the robot as well as finding the robot less capable of adapting to their needs, less useful and less enjoyable (see Table 2). Robot familiarity was not found to significantly correlate with any other constructs within the Almere questionnaire. In relation to robot perception, robot familiarity also negatively correlated with robot likeability $(r=-0.41, n=45, p=$ .01 ), with the robot perceived as less likeable as familiarity increased. Robot familiarity was not found to significantly correlate with any other constructs within the Godspeed questionnaire.
Table 1: Participant Ratings of Robot Familiarity.

\begin{tabular}{lll}
\hline & Frequency & Percent \\
\hline Somewhat familiar & 34 & 39.53 \\
Undecided & 11 & 12.79 \\
Not very familiar & 28 & 32.56 \\
Not at all familiar & 13 & 15.12 \\
Total & 86 & 100.00 \\
\hline
\end{tabular}

\subsection{User mood and social robot acceptance}

Prior to investigating the relationship between participant mood and robot acceptance correlation analyses were conducted to examine any interactions both within and across the two mood measures. Firstly a correlation analysis was conducted between participants' explicit positive affect and explicit negative affect, as measured by the PANAS-X. Positive affect moderately and negatively correlated with negative affect ( $r=-.33, n=45, p=.03)$, with positive affect increasing as negative affect decreased. The relationship between implicit positive affect and implicit negative affect, as measured by the iPANAT was also examined. This also found a strong correlation $(r=0.79, n=45, p<.001)$; however, this relationship was positive with positive affect increasing as negative affect also increased. Implicit mood and explicit mood did not correlate across any of the four dimensions (implicit/explicit, positive/negative).

Correlation analyses were then carried out to examine the relationship between explicit and implicit mood and the 12 constructs included in the Almere questionnaire (see Table 3). Explicit positive affect moderately and negatively correlated with perceived usefulness $(r=-0.31)$, trust $(r=-0.41)$ and social influence $(r=-0.35)$ with those feeling more sleepy and sluggish finding the robot more useful, were more trusting of it but were more considerate of the opinions of others in relation to the robot. Explicit negative affect moderately and positively correlated with both social influence $(r=0.46)$ and perceived enjoyment in a robot $(r=0.37)$ with increased negative affect resulting in more perceived enjoyment but more concern over the opinions of others. Correlation analyses were also conducted between implicit positive affect and acceptance finding significant correlations with both facilitating conditions $(r=$ $0.40)$ and intention to use $(r=0.42)$ and a weak relationship with perceived enjoyment $(r=.29)$. All relationships were positive with participants experiencing implicit negative affect more likely to feel they had the right tools to use the robot, were more likely to use it and more likely to enjoy using it. Anxiety using the robot $(r=.05)$ and intention 
Table 2: Significant Correlations Between Robot Familiarity and Robot Acceptance.

\begin{tabular}{llllllll}
\hline & & $\begin{array}{l}\text { Facilitating } \\
\text { conditions }\end{array}$ & $\begin{array}{l}\text { Intention } \\
\text { to use }\end{array}$ & $\begin{array}{l}\text { Perceived } \\
\text { adaptability }\end{array}$ & $\begin{array}{l}\text { Perceived } \\
\text { enjoyment }\end{array}$ & $\begin{array}{l}\text { Perceived } \\
\text { sociability }\end{array}$ & $\begin{array}{l}\text { Perceived } \\
\text { usefulness }\end{array}$ \\
\hline $\begin{array}{l}\text { Robot } \\
\text { familiarity }\end{array}$ & $\begin{array}{l}\text { Pearson } \\
\text { correlation } \\
\text { Sig. } \\
(2 \text {-tailed })\end{array}$ & -.50 & -.40 & -.36 & -.37 & -.52 & .38 \\
& $<.001$ & .01 & .01 & .01 & $<.001$ & .01 \\
\hline
\end{tabular}

to use the robot $(r=.05)$ correlated with implicit negative affect, however these relationships were weak.

Finally, correlation analyses investigated the relationship between mood and the Godspeed questionnaire with significant relationships found only in relation to explicit negative affect. Explicit negative affect positively correlated with the construct of intelligence ( $r=0.36, n=45, p$ $=.01$ ) but negatively correlated with perceived robot safety $(r=-0.42, n=45, p<.01)$. Therefore, those feeling more distressed or fearful perceived the robot to be more intelligent but less safe. All other relationships between mood and robot perception were non-significant.

\subsection{The Almere acceptance questionnaire and the Godspeed perception questionnaire}

The Almere questionnaire and the Godspeed questionnaire were additionally analysed to better understand how they relate to each other. Moderate positive correlations were found between the Godspeed's 'perceived robot intelligence' component and the Almere's 'attitude towards technology' $(r=0.33, n=45, p=.03)$, 'facilitating conditions' $(r=0.33, n=45, p=.03)$ and social influence $(r=0.37$, $n=45, p=.01)$ constructs. Therefore, participants perceiving a robot as more intelligent also had a more positive attitude towards the technology, felt they had the right tools and knowledge to use the technology but required support from family and friends when deciding whether to use it.

\subsection{Robot displayed emotion and acceptance}

A one way analysis of variance was undertaken to investigate the influence displayed robot emotion has on the 12 constructs in the Almere questionnaire of robot acceptance with no significant results found. Further analyses were then conducted on the individual questions found under each construct of the Almere questionnaire. Within the anxiety construct a significant difference was found in ratings of the question 'If I use the robot, I would be afraid to break something' across the three experimental levels $\left(F(2,59)=5.66, p<.01 \eta_{p}^{2}=.16\right)$. Post-hoc Tukey analysis found the positive robot $(M=3.80, S D=1.11)$ to be rated significantly higher on this question than both the negative $(M=3.00, S D=1.18) p=.04$, and neutral $(M=3.24$, $S D=1.45) p<.01$ robot suggesting participants perceive the positive robot easier to break. Within the perceived adaptiveness construct, the question 'I think the robot will help me when I consider it necessary' was also found to be significantly different depending on experimental level $(F(2$, 59) $\left.=3.79, p=.03 \eta_{p}^{2}=0.11\right)$. Post-hoc Tukey analysis found the neutral robot $(M=2.81, S D=1.33)$ to be perceived as significantly less helpful than the positive robot $(M=3.60$, $S D=0.94) p=.05$ with a non -significant trend suggesting the negative robot $(M=3.57, S D=0.81) p=.06$ also more helpful than the neutral robot. No other questions within the perceived adaptiveness construct significantly differed across experimental levels.

Finally, a significant difference was found in the question 'I would find the robot easy to use' $(F(2,59)=3.88, p$ $\left.=.03, \eta_{p}^{2}=0.12\right)$ with the negative robot $(M=3.52, S D 0.18)$ seen as significantly easier to use than the positive robot $(M=2.75, S D=0.91) p=.03$. A significant difference was also found for the question 'I think I could use the robot when I have a good manual' $\left(F(2,59)=4.02, p=.02 \eta_{p}^{2}=\right.$ 0.12) with Tukey analysis indicating the positive robot $(M$ $=3.36, S D=0.93)$ as potentially easier to use with a manual than the negative robot $(M=2.33, S D=1.39) p=.03$.

A one way analysis of variance was also conducted to investigate the impact robot displayed emotion has on robot perception, as measured by the Godspeed questionnaire. Again, no significant differences were highlighted in the overarching constructs; however, further analysis of the questions within the questionnaire indicate that on the 'Foolish-Sensitive' semantic scale a significant difference was found $\left(F(2,59)=4.78, p=.01 \eta_{p}^{2}=0.14\right)$, with the positive robot $(M=3.40, S D=0.68)$ perceived as signif- 
Table 3: Significant Correlations Between User Mood and Robot Acceptance.

\begin{tabular}{|c|c|c|c|c|c|c|c|c|c|c|c|c|c|}
\hline & & ANX & ATT & FC & ITU & PAD & PENJ & PEOU & PS & PU & SI & SP & Trust \\
\hline PANAS & Corr. & -.06 & -.16 & -.20 & -.10 & -.16 & -.03 & -.04 & .14 & -.31 & -.35 & .08 & -.41 \\
\hline PA & Sig. & .71 & .29 & .18 & .49 & .29 & .87 & .82 & .38 & $.04^{\star}$ & $.02^{\star}$ & .63 & $.01^{*}$ \\
\hline PANAS & Corr. & -.90 & .23 & .12 & .25 & -.09 & .37 & .17 & .03 & .28 & .46 & .04 & .06 \\
\hline NA & Sig. & .55 & .13 & .42 & .10 & .54 & $.01^{\star}$ & .28 & .84 & .06 & $<.01^{\star \star}$ & .78 & .71 \\
\hline IPANAT & Corr. & .18 & .21 & .40 & .42 & .14 & .29 & .19 & .29 & .23 & .23 & .22 & .28 \\
\hline PA & Sig. & .23 & .17 & $.01^{\star}$ & $<.01^{\star \star}$ & .36 & .05 & .21 & .06 & .14 & .12 & .15 & .07 \\
\hline IPANAT & Corr. & .29 & .17 & .25 & .29 & .11 & .28 & .06 & .17 & .23 & .20 & .12 & .28 \\
\hline NA & Sig. & .05 & .25 & .10 & .05 & .49 & .06 & .71 & .27 & .13 & .19 & .42 & .06 \\
\hline$N=45$ & & & & & & & & & & & & & \\
\hline
\end{tabular}

icantly more foolish than both the neutral $(M=4.05, S D$ $=0.86) p=.02$ and negative $(M=3.95, S D=0.13)$ robot. No other questions significantly differed between experimental levels. In order to investigate the influence a participant's mood (sad/happy) has on the acceptance of either a positive, negative or neutral robot a $2 \times 3$ analysis of variance was conducted. This analysis yielded a significant interaction between robot emotion and mood on the construct of social influence $\left(F(2,38)=5.58, p=.01, \eta_{p}^{2}=\right.$ $0.23)$, with sad participants rating social influence as less important when using a negative robot $(M=2.25, S D=0.97)$ than happy participants $(M=3.50, S D=0.97)$.

\section{Discussion}

This study investigated whether the acceptance of socially assistive robots and the way in which they are perceived is influenced by both user characteristics and characteristics displayed by the robot. Previous research has largely focused on user characteristics or robot characteristics only, with the goal of this paper being to study these combined influences within a single experiment. Findings from this research suggest that gender and education may not be associated with acceptance; however, several constructs of the acceptance questionnaire did significantly correlate with age and mood. As an example, those younger and those experiencing sadness or loneliness were more dependent on the opinions of others (as measured by the social influence construct of the acceptance questionnaire) when deciding whether to use the robot. This highlights the potential benefit of family and friends in the integration of social robots into vulnerable populations. Robot fa- miliarity also correlated with robot acceptance with those more familiar finding the robot less useful and less enjoyable, this is important as robots become more prominent in society. Displayed robot emotion significantly influenced acceptance and perception with the positive robot appearing more childlike than the negative and neutral robot, and the neutral robot the least helpful. These findings emphasise the importance of taking into account both user and robot characteristics in the successful integration of social robots.

\subsection{User characteristics and social robot acceptance}

In order to determine how user characteristics relate to the acceptance of social robots age, gender, level of education and robot familiarity were analysed with significant correlations predicted within each characteristic. It was firstly predicted that acceptance would correlate with age, with Heerink's [15] study on older adults finding a significant relationship between age and the constructs of robot anxiety and intention to use. These findings were not replicated during this study, when a wider age range was utilised. Older adults were however found less likely to see a social robot interaction as pleasurable, potentially leading to robot disuse. It is therefore important for innovators to consider elements within social robots that may increase the fun and enjoyment experienced by older adults. Conversely, younger users were found to be more influenced by the opinions of their social network highlighting the importance of including family and friends in the integration of a social robot should users be younger in age. These findings are a novel addition to robotics research as 
previous studies around age and acceptance have focused largely on functional aspects ignoring these more social factors.

Gender had also previously been found to relate to robot acceptance with males finding a robot easier to use than females and females being more anxious about using the robot than men [17], [15]. These differences were again not supported during this study; however, Heerink focused on participants aged 65 years plus suggesting a gender divide more prevalent in the older adult population with younger generations potentially more equal in respect of their confidence in using technology. It is however important to note that around three quarters of the participants included in this study were female, with different results possible should more males have taken part. Participant education was also previously found to relate to robot anxiety and perceived sociability in the older adult population [15]. These findings were again not replicated during this study; however, specific details on participant education were not given within Heerink's paper making it difficult to directly compare these results. Participants within this particular research were a relatively welleducated sample with only $4 \%$ in receipt of GCSEs only, and around $50 \%$ of participants having worked towards an undergraduate degree. Should this study be replicated with a more diverse sample it is possible that different results will be found.

Robot familiarity was found to have the largest influence on the human-robot interaction with five constructs of acceptance and two constructs of robot perception relating to this characteristic. All of these constructs negatively correlate suggesting higher robot familiarity to have a negative impact on an individual's attitudes and behaviours toward a social robot, of high importance as society moves towards a more robot integrated world. Heerink [15], when analysing computer experience, found it to correlate with facilitating conditions, anxiety and perceived ease of use suggesting those more experienced in computers felt better equipped and more capable of using the robot. This current study found facilitating conditions to negatively correlate with robot familiarity, with those more familiar feeling less confident they had the knowledge and tools required to use it. Heerink's study did however focus on 'computer experience' as opposed to 'robot familiarity' which whilst similar may have resulted in differing results. Robot familiarity may be viewed more as a person's awareness of robots, whereas computer experience may be viewed as a person's direct interaction with technology more generally.

Those more familiar with robots also felt the robot was less able to adapt to their needs, was less useful to them and left them less intent on using it. This direct sequence of constructs is found within the Almere model itself, working linearly towards robot use or disuse. These findings not only provide support for this particular process within the Almere model but also highlight the considerable influence robot familiarity can have on the humanrobot interaction. Those more familiar also perceived the robot as less likeable and less enjoyable to interact with. A robot that appears neither useful nor fun will be less likely to motivate a user to invest their time. Finally, those more familiar with robots also found the robot less safe, potentially due to increased knowledge around the robots limited capabilities. Together these findings suggest that increased robot familiarity can result in a heightened awareness of a robot's limitations - both as an assistive robot and a social entity. This is crucial in a society where robots are being further integrated each year.

\subsection{User mood and social robots}

In addition to participant characteristics, mood was investigated to better understand its relationship with the way in which a robot is accepted. This is of particular importance when socially assistive robots are assigned to improve an individual's general well-being. Whilst research in relation to mood and robot acceptance is limited, Baisch et al. [21] did find life satisfaction to positively correlate with intention to use a robot, although a relationship with depressive mood was not found. Within this study participant mood significantly related to acceptance across a number of constructs. Firstly, those experiencing high explicit positive affect and therefore subjective feelings of excitement and enthusiasm viewed a robot as less useful then those feeling more sleepy and sluggish. It is possible that this is due to those more excited and enthusiastic feeling better able to undertake the tasks themselves, and those more sluggish anticipating the need for more help, potentially resulting in misuse of a robot and over reliance. Trust was also lower in those experiencing high positive affect, leaving an excited and enthusiastic user even more willing to undertake the tasks themselves. However, those experiencing high implicit positive affect felt more confident they had the knowledge and tools required to use the robot and were more intent on using it. Explicit and implicit positive affect were not found to significantly correlate in this study suggesting feelings of excitement or drowsiness often go unnoticed or undeclared. It is also possible that the iPANAT is more a measure of unconscious and automatic arousal rather than conscious valance [45]. Bringing together results from both of these measures it 
appears that whilst those feeling more subjectively excited or enthusiastic do not necessarily overtly see the benefits of using a social robot, high implicit affect, or arousal, will result in an individual being more likely to commit to using the robot long term.

Both low explicit positive affect and high explicit negative affect were also found to result in heavier reliance on others opinions when deciding whether or not to use the robot. When these specific affects are experienced together the result is that of sadness or loneliness [25]. This suggests that users experiencing low mood may be more easily influenced by the opinions of others whether these opinions are positive or negative in nature. These findings again highlight the potential benefit of involving family and friends in the integration of a socially assistive robot, specifically when the user is from a vulnerable population.

It was also found that those experiencing more subjective negative affect potentially due to distress, nervousness or hostility, viewed the robot as more enjoyable to use in addition to perceiving it as more intelligent. Perceived robot intelligence, a construct within the Godspeed questionnaire, was found to significantly correlate with acceptance suggesting that those with high negative affect will have higher robot acceptance. Those with higher negative affect did however also perceive the robot as less safe which could directly result in robot disuse irrespective of their views. Taken together these findings support the hypothesis that user mood significantly relates to robot acceptance and that user mood will likely be reflected on to attitudes and behaviours towards the robot. Mood should therefore be considered when looking to integrate socially assistive robots, particularly into clinical settings.

\subsection{Robot displayed emotion and acceptance}

Emotional exchange is an important aspect of humanhuman social interaction [27], with an individual suppressing emotion impacting the ability to form a bond [28]. Research on what a user expects from their social robot also supports the idea that it should look to express emotion, particularly empathy [6], [29]. This research therefore investigated whether a robot displaying positive, negative or neutral emotion impacted how the robot was accepted or perceived. Robot displayed emotion did not significantly influence any of the overarching constructs found within either of the questionnaires, suggesting that people's acceptance of the robot was not influenced by its expressed emotion. It is however important to note that the emotions displayed whilst contextual were not directed specifically toward the participant and therefore cannot be likened to other-oriented emotion such as empathy. The robot emotion was also only displayed for a short period of time and on its own, potentially downplaying its significance within a longer interaction. Future research should investigate whether the same is found when the robot's emotions are directly related to the participant, are present over a longer period of time and also when they appear during a generally neutral conversation.

The positive robot was perceived as significantly more foolish than the negative and neutral robot as well as easier to break. The higher speed and tone used for the positive robot's voice made it possible that participants viewed the robot as childlike. These findings are important to consider when programming happy emotion into a robot, specifically if the robot needs to appear serious and capable as in the case of SAR. The neutral robot was perceived as significantly less 'able to help when considered necessary' than the positive and negative robot. This is potentially due to its tone and manner appearing less socially flexible, although other adaptability questions were not affected and therefore further investigations into this question are required. Finally, whilst the negative robot was rated significantly easier to use than the positive robot, this difference switched when a user manual was said to be available. This suggests that the positive robot is seen as a more complicated machine but that with help it becomes much easier to work with than the negative robot.

Interaction analyses between a user's mood and a robot displayed emotion found a significant difference in the construct of social influence. Contrary to the previous findings that sad participants relied more on the opinion of others when interacting with a neutral robot, when presented with a negative robot sad participants were found to be less influenced by the opinions of others. This suggests that user mood may affect social influence differently depending on displayed robot emotion, however this result lacked power due to low numbers of participants in the 'sad' group; Future studies are needed to investigate this further.

\subsection{Limitations}

This study utilised videos of a social robot as opposed to participants having access to a real robot. Whilst this allowed for a larger sample size, findings could have been different had the embodiment of a robot been available to the sample. Whilst the paper has a relatively high number of participants, participants included in the 'happy' and 'sad' groups when split by robot emotion were low, future 
research should look to replicate this part of the study with higher numbers. It is also important to note that around two thirds of the sample were recruited via a university participant pool which may not be representative of populations requiring SAR. The robot featured in the videos was a Pepper robot, which has many different qualities than other forms of SAR. Should a different robot have been used in the study alternative results may have been found. Finally, whilst storytelling may be one task undertaken by SAR there are many other tasks in which SAR may be used. Use of an alternative task may have resulted in different findings.

\section{Conclusion and future research}

With people living longer, the additional pressures experienced by healthcare systems and a patient's family and friends has increased the priority of integrating socially assistive robots into homes, hospitals and care homes. This study investigated the potential influence both user characteristics and robot emotion can have on the acceptance of socially assistive robots, in order to highlight variables within the user and the robot that should be considered when looking to develop and integrate the technology. A number of factors were identified that present challenges when looking to integrate SAR; particularly into vulnerable populations such as older adults and those potentially experiencing low mood, loneliness or distress. The results of this study suggest that the inclusion of a patient's family and friends at the point of integration is important to ensure that everyone is clear on the benefits of the robot for continued use. As robots become more integrated into society it is also important to be aware of the impact increased robot familiarity can have on robot acceptance. Increased robot familiarity can reduce an individual's views on how useful and enjoyable the robot appears requiring innovators to build capabilities and applications into the robot to counter this challenge as technology moves forward. Finally, a robot's display of positive emotion may need to be limited in order to appear less childlike and more robust. This paper therefore found that human characteristics, robot characteristics, and their potential influence on each other can affect social robot acceptance.

Future research should continue to investigate the influence displayed robot emotion has across a longer time period, with a more user-orientated focus, and when introduced during a generally neutral conversation. It is also important to further investigate whether the acceptance of a positive, negative or neutral robot differs when a user is experiencing different moods. This research highlights the importance for roboticists, innovators and integrators of socially assistive robots to consider both user and robot characteristics when looking to achieve successful integration, particularly within vulnerable populations.

Acknowledgements: We would like to thank all participants who participated in our experiment and Caroline Brown for her useful feedback.

Funding: This work has received funding from the European Union's Horizon 2020 research and innovation programme under the Marie Skłodowska-Curie grant agreement No 721619 for the SOCRATES project.

\section{References}

[1] A. Winfield, Robotics: A very short introduction, OUP Oxford, 2012

[2] W. H. Organization, World report on ageing and health, World Health Organization, 2015

[3] S. M. S. Khaksar, R. Khosla, M. T. Chu, F. S. Shahmehr, Service innovation using social robot to reduce social vulnerability among older people in residential care facilities, Technological Forecasting and Social Change, 2016, 113, 438-453

[4] P. Khosravi, A. Rezvani, A. Wiewiora, The impact of technology on older adults' social isolation, Computers in Human Behavior, 2016, 63, 594-603

[5] D. Feil-Seifer, M. J. Mataric, Defining socially assistive robotics, In: 9th International Conference on Rehabilitation Robotics (ICORR 2005), IEEE, 2005, 465-468

[6] M. M. de Graaf, S. B. Allouch, J. van Dijk, What makes robots social?: A user's perspective on characteristics for social human-robot interaction, In: International Conference on Social Robotics, Springer, 2015, 184-193

[7] H. Robinson, B. MacDonald, E. Broadbent, The role of healthcare robots for older people at home: A review, International Journal of Social Robotics, 2014, 6(4), 575-591

[8] S. M. Rabbitt, A. E. Kazdin, B. Scassellati, Integrating socially assistive robotics into mental healthcare interventions: Applications and recommendations for expanded use, Clinical Psychology Review, 2015, 35, 35-46

[9] N. Ezer, A. D. Fisk, W. A. Rogers, Attitudinal and intentional acceptance of domestic robots by younger and older adults, In: International Conference on Universal Access in Human-Computer Interaction, Springer, 2009, 39-48

[10] C.-A. Smarr, et al., Domestic robots for older adults: attitudes, preferences, and potential, International Journal of Social Robotics, 2014, 6(2), 229-247

[11] J. M. Beer, A. Prakash, T. L. Mitzner, W. A. Rogers, Understanding robot acceptance, Technical report, Georgia Institute of Technology, 2011

[12] V. Venkatesh, M. G. Morris, G. B. Davis, F. D. Davis, User acceptance of information technology: Toward a unified view. MIS 
Quarterly, 2003, 425-478

[13] I. Gaudiello, E. Zibetti, S. Lefort, M. Chetouani, S. Ivaldi, Trust as indicator of robot functional and social acceptance, An experimental study on user conformation to iCub answers, Computers in Human Behavior, 2016, 61, 633-655

[14] M. Heerink, B. Kröse, V. Evers, B. Wielinga, Assessing acceptance of assistive social agent technology by older adults: the Almere model, International Journal of Social Robotics, 2010, 2(4), 361-375

[15] M. Heerink, Exploring the influence of age, gender, education and computer experience on robot acceptance by older adults, In: Proceedings of the 6th International Conference on HumanRobot Interaction, ACM, 2011, 147-148

[16] I. H. Kuo, et al., Age and gender factors in user acceptance of healthcare robots, In: 2015 24th IEEE International Symposium on Robot and Human Interactive Communication (ROMAN), IEEE, 2009, 214-219

[17] M. M. De Graaf, S. B. Allouch, Exploring influencing variables for the acceptance of social robots, Robotics and Autonomous Systems, 2013, 61(12), 1476-1486

[18] K. O. Arras, D. Cerqui, Do we want to share our lives and bodies with robots? A 2000-people survey, Technical Report, 2005

[19] A. Weiss, C. Bartneck, Meta analysis of the usage of the Godspeed Questionnaire Series, In: RO-MAN 2015 - The 24th IEEE International Symposium on Robot and Human Interactive Communication, IEEE, 2015, 381-388

[20] N. Schwarz, G. L. Clore, Mood, misattribution, and judgments of well-being: informative and directive functions of affective states, Journal of personality and social psychology, 1983, 45(3), 513-523

[21] S. Baisch, T. Kolling, M. Knopf, Factors impacting on older and younger peoples' perceptions of elderly robot users, Innovation in Aging, 2017, Suppl 1, 1190-1190

[22] I. Blanchette, A. Richards, The influence of affect on higher level cognition: A review of research on interpretation, judgement, decision making and reasoning, Cognition and Emotion, 2010, 24(4), 561-595

[23] C. Beedie, P. Terry, A. Lane, Distinctions between emotion and mood, Cognition and Emotion, 2005, 19(6), 847-878

[24] D. Watson, L. A. Clark, A. Tellegen, Development and validation of brief measures of positive and negative affect: the PANAS scales, Journal of personality and social psychology, 1988, 54(6), 1063-1070

[25] D. Watson, L. A. Clark, The PANAS-X: Manual for the positive and negative affect schedule-expanded form, lowa Research Online, 1999

[26] M. Quirin, M. Kazén, J. Kuhl, When nonsense sounds happy or helpless: the implicit positive and negative affect test (IPANAT), Journal of Personality and Social Psychology, 2009, 97(3), 500516

[27] D. Keltner, J. Haidt, Social functions of emotions at four levels of analysis, Cognition and Emotion, 1999, 13(5), 505-521

[28] E. A. Butler, B. Egloff, F. H. Wlhelm, N. C. Smith, E. A. Erickson, J. J. Gross, The social consequences of expressive suppression, Emotion, 2003, 3(1), 48-67

[29] S. Brave, C. Nass, K. Hutchinson, Computers that care: investigating the effects of orientation of emotion exhibited by an embodied computer agent, International Journal of HumanComputer Studies, 2005, 62(2), 161-178
[30] I. Leite, A. Pereira, S. Mascarenhas, C. Martinho, R. Prada, A. Paiva, The influence of empathy in human-robot relations, International Journal of Human-Computer Studies, 2013, 71(3), 250260

[31] A. Paiva, I. Leite, H. Boukricha, I. Wachsmuth, Empathy in virtual agents and robots: a survey, ACM Transactions on Interactive Intelligent Systems (TiiS), 2017, 7(3), Article 11

[32] S. Chumkamon, E. Hayashi, M. Koike, Intelligent emotion and behavior based on topological consciousness and adaptive resonance theory in a companion robot, Biologically Inspired Cognitive Architectures, 2016, 18, 51-67

[33] A. Dijksterhuis, J. A. Bargh, The perception-behavior expressway: Automatic effects of social perception on social behaviour, In: M. P. Zanna (Ed.), Advances in Experimental Social Psychology, San Diego, CA, US: Academic Press, 2001, 33, 1-40

[34] M. R. Banaji, C. Hardin, A. J. Rothman, Implicit stereotyping in person judgment, Journal of Personality and Social Psychology, 1993, 65(2), 272-281

[35] M. L. Walters, The design space for robot appearance and behaviour for social robot companions, Ph.D. thesis, 2008

[36] J. Goetz, S. Kiesler, Cooperation with a robotic assistant, In: CHl'02 Extended Abstracts on Human Factors in Computing Systems (ACM, 2002) 578-579

[37] J. Goetz, S. Kiesler, A. Powers, Matching robot appearance and behavior to tasks to improve human-robot cooperation, In: Proceedings of the 12th IEEE International Workshop on Robot and Human Interactive Communication, IEEE Press Piscataway, NJ, 2003, 55-60

[38] B. R. Duffy, Anthropomorphism and the social robot, Robotics and autonomous systems, 2003, 42(3-4), 177-190

[39] C. Bartneck, D. Kulić, E. Croft, S. Zoghbi, Measurement instruments for the anthropomorphism, animacy, likeability, perceived intelligence, and perceived safety of robots, International Journal of Social Robotics, 2009, 1(1), 71-81

[40] M. Mori, The uncanny valley, Energy, 1970, 7(4), 33-35

[41] S. Baisch, et al., Acceptance of social robots by elder people: does psychosocial functioning matter?, International Journal of Social Robotics, 2017, 9(2), 293-307

[42] A. Beck, et al., Interpretation of emotional body language displayed by a humanoid robot: A case study with children, International Journal of Social Robotics, 2013, 5(3), 325-334

[43] D.-S. Kwon, et al., Emotion interaction system for a service robot, In: RO-MAN 2007 - The 16th IEEE International Symposium on Robot and Human Interactive Communication, IEEE, 2007, 351-356

[44] A. van Maris, N. Zook, P. Caleb-Solly, M. Studley, A. Winfield, S. Dogramadzi, Ethical considerations of (contextually) affective robot behaviour, (forthcoming)

[45] K. R. Scherer, What are emotions? And how can they be measured?, Social Science Information, 2005, 44(4), 695-729 